\title{
Mediterranee comme frontiere : ordre symbolique, materialisation des corps et immigration
}

O mediterrâneo como fronteira: ordem simbólica, materialização dos corpos e imigração.

Mediterranean as a frontier: symbolic order, body materialization and migration

Mediterráneo como frontera: orden simbólica, materialización de los cuerpos y migración

\section{Caterina Rea}

Université de Lille, França.

\section{Resumo}

Quais são as dinâmicas sociais, políticas e psicológicas que caracterizam as relações NorteSul, entre Oriente-Ocidente? Este texto analisa a realidade do Mediterrâneo como espaço histórico-cultural onde estas dinâmicas operam segundo eixos diferentes. O Mediterrâneo é uma fronteira aberta, um cruzamento de culturas, mas também - como mostram os estudos pós-coloniais - um lugar de separação atravessado por lógicas de dominação e de poder. As categorias política-filosóficas de desnaturalização e instituição se revelam aqui como operadores fundamentais de uma leitura crítica do espaço mediterrâneo, particularmente do exemplo pós-colonial francês.

Palavras Chave: Corpo, Desnaturalização, Instituição, Poder, (Pós) Colonização.

\begin{abstract}
Which are the social, political and psychological dynamics that characterize north-south and east-west relations? This text analyses the Mediterranean as a cultural and historical territory where these dynamics operate following different axes. The Mediterranean is an open frontier, a crossroad of cultures, but also - as described by post-colonial studies - a dividing border crossed by domination and power logics. The philosophical and political concepts of denaturalization and institution are used here as fundamental categories for a critical analysis of Mediterranean space and particularly of the French (post) colonial case.
\end{abstract}

Keywords: Body, Denaturalization, Institution, Post Colonization.

\section{Resumen}

¿Cuales son las dinámicas sociales, políticas y psicológicas que caracterizan las relaciones entre Norte y Sur, entre Oriente y Occidente? Este texto analiza la realidad del Mediterráneo 
como espacio histórico-cultural donde estas dinámicas operan según ejes diferentes. El Mediterráneo es una frontera abierta, un cruce de culturas, pero también - como muestran los estudios pos-coloniales - un lugar de separación atravesado de lógicas de dominación y de poder. Las categorías político-filosóficas de desnaturalización y de institución se revelan aquí como operadores fundamentales de una lectura crítica del espacio mediterráneo y particularmente del ejemplo (pos)-colonial francés.

Palabras clave: Cuerpo, Desnaturalización, Institución, Poder, (Post) Colonización.

\section{Résumé:}

Quelles sont les dynamiques sociales, politiques et psychologiques qui caractérisent les relations entre Nord et Sud, entre Orient et Occident? Ce texte analyse la réalité de la Méditerranée en tant qu'espace historico-culturel ou' ces dynamiques opèrent selon des lignes directrices différentes. La Méditerranée est une frontière ouverte, un entrelacement de cultures, mis aussi - comme le montrent bien les études postcoloniales- un lieu de séparation, traversé par des logiques de domination et de pouvoir. Les catégories politico-philosophiques de dénaturalisation et d'institution se révèlent ici comme les opérateurs fondamentaux d'une lecture critique de l'espace méditerranéen et particulièrement de l'exemple (post)-colonial français.

Mots clés: Corps, Dénaturalisation, Institution, Pouvoir, (Post)-Colonisation.

Selon une conception assez répandue, qui a été celle défendue par le «Centro Braudel» de l'Université de Catane lors du colloque international sur la Méditerranée, celle-ci incarne le lieu primaire de médiation et de rencontre entre des cultures, des histoires et des peuples différents. Selon cette lecture, la Méditerranée apparaît comme un lieu de «contamination», comme une frontière poreuse qui permet l'échange de traditions, de paradigmes interprétatifs et d'univers symboliques divers. La Méditerranée est, lit-on dans les propos du colloque - « une histoire de dialogue et de coexistence millénaire entre des mondes divers et contigus, grâce à laquelle nous pourrions dire qu'elle s'est toujours constituée comme l'élément de la médiation ${ }^{1}$. Ainsi, elle deviendrait l'emblème de cette identité ouverte et plurielle qui donne sens au projet européen lui-même. «Tel est le langage de l'occident et, en ce sens l'identité de l'Europe est constituée par l'absence d'identité rigide; à partir de l'histoire de la Méditerranée, en effet, l'identité de l'Europe se constitue comme identité d'accueil $»^{2}$. Nous ne pouvons 
que partager ces intentions riches d'éléments féconds et animées d'une forte charge humaniste et de l'effort de faire dialoguer les cultures et les traditions qui constituent l'espace méditerranéen. Néanmoins, une telle lecture ne devraitelle pas être mise à l'épreuve d'une autre perspective, peut-être moins optimiste et positive, à l'égard de ce que la Méditerranée a incarné et incarne encore ? Ce texte prendra en compte le point de vue des études post-coloniales qui forment aujourd'hui une branche en expansion, avec les questions de genre et les études culturelles $^{3}$. Le tableau qu'une telle approche dresse à propos de la Méditerranée apparaît quelque peu noirci, car elle y voit l'espace où se sont joués et où continuent de se jouer des rapports de dominations, des inégalités relatives au système colonial et à son héritage encore d'actualité. Comme le rappelle R. Ivekovic dans son intervention au même colloque de Catane, l'espace méditerranéen a toujours été le lieu de partage et de division entre «le propre et l'étrange, l'Europe et les autres. La Méditerranée n'est jamais restée en dehors de ces divisions, voire elle y a toujours participé $»^{4}$. Depuis la colonisation avec l'exaltation du prétendu rationalisme européen, devenu l'instrument d'une 'légitimation' des violences envers les colonisés, du refus de liberté et de citoyenneté à leur égard, jusqu'à la tragédie actuelle de beaucoup de migrants, le mare nostrum est traversé par bien des asymétries et des hiérarchies. Dès lors, comme le dit encore R. Ivekovic, « nous ne pouvons plus nous contenter de son image idéale (même parce que dans l'histoire des deux côtés de la mer, il y a la responsabilité de l'Europe) $»^{5}$.

Nous ne songeons pas à opposer ces deux lectures qui nous semblent bien partager un point à notre avis crucial : la primauté historique de l'expérience culturelle de la Méditerranée et la dénaturalisation de toute dimension sociale voire même identitaire. Contre l'affirmation des revendications identitaires qui rendent rigides les phénomènes sociaux en les transformant en prétendus donnés naturels, il s'agira à tout moment de revendiquer «l'insistance sur l'historicité de la dimension culturelle de la Méditerranée ${ }^{6}$. En renonçant à toute lecture de la Méditerranée en termes identitaires, prétendument capables de définir son espace originaire et son destin, il s'agira de souligner plutôt ses multiples altérations historiques.

Cela signifie que la Méditerranée, car elle est frontière, ne peut avoir aucune essence originaire, aucune nature intime, aucun destin qui lui serait propre : le seul contenu capable de donner une configuration concrète à la frontière méditerranéenne est ce qui à chaque fois 
parvient à en faire la concrétude factuelle des processus historiques ${ }^{7}$.

En proposant cette lecture dénaturalisée de la Méditerranée en tant que contestation de toute frontière identitaire ou de sang, il ne faudra bien évidemment pas oublier qu'une barrière racialisée et de sang a le plus souvent été imposée dans la Méditerranée par l'histoire coloniale et par son lourd héritage dont on a du mal à se débarrasser. Dans ce contexte, la Méditerranée a été (et elle est souvent encore) instituée comme une frontière qui marque les corps de ceux qui l'habitent en les matérialisant et en les produisant selon des rapports de pouvoirs différents. Je pense ici en particulier aux analyses de Sidi Mohammed Barkat sur l'état de droit colonial en tant que dispositif de marquage et de production de corps, ceux des « indigènes », dont le statut même d'humain n'est pas pleinement reconnu comme étant réalisé. Dans l'institution de ces « corps d'exception » se joue ce que Barkat appelle justement « une métaphysique de 1 'histoire $»^{8}$ avec ses dogmes et ses logiques naturalisées qui puisent dans une rhétorique politique des origines. En effet, il faut bien ici que « les générations qui se succèdent se convainquent de l'idée selon laquelle la vérité ou l'authenticité de la nation dépend principalement de l'origine de ses membres. Le colonialisme, l'état de droit colonial, a porté loin cette idée de l'origine $»^{9}$.

En reprenant l'idée de dénaturalisation comme clé de lecture de l'histoire culturelle de l'espace méditerranéen, nous n'oublions pas que le recours à une logique de la naturalisation a souvent été une stratégie politique et un instrument idéologique pour défendre et garder certains rapports de pouvoir. Comme le dit Judith Butler, « le recours au naturel est toujours politique $»^{10}$. Elle se réfère en particulier à la logique patriarcale et à l'institution de rapports de domination en fonction du genre, mais il nous semble bien qu'une telle logique s'étend à d'autres sphères des rapports de pouvoir. En effet, plus généralement, « la construction politique du sujet se fait à des fins précises de légitimation et d'exclusion, et ces processus politiques se trouvent effectivement masqués et naturalisés par toute analyse politique qui les fonderait dans les structures juridiques $»^{11}$. Et c'est bien à ces logiques discursives de domination que nous devons nous atteler pour comprendre leur fonctionnement au sein de l'espace méditerranéen.

\section{Méditerranée d'exception : colonisation et authenticité de la nation}

Ce texte vise ainsi à interroger la perspective culturelle qui voit dans la 
Méditerranée une sorte de 'visage humaniste' de l'Europe, c'est-à-dire le lieu de son héritage classique, euroméditerranéen justement, qui ferait contrepoint au modèle d'une Europe économique qui risque de plus en plus d'être résorbée par les logiques du marché et de la globalisation. La Méditerranée devient le modèle d'intégration à laquelle l'Europe ne peut que rester liée afin de promouvoir l'union entre les peuples. L'intérêt indubitable d'une telle perspective consiste dans l'affirmation que le sens même, historique et culturel, de l'Europe ne peut pas faire l'impasse de son propre décentrement, à savoir de la référence à son âme méditerranéenne, méridionale ou même orientale, donc d'une pluralité de composantes qui forment et façonnent son identité complexe et multiple. Comme le rappelle à ce propos le philosophe et juriste Pietro Barcellona, ce qui est essentiel c'est la tâche de délinéer un horizon dans lequel le Sud ne se situe pas dans une position résiduelle, en marge d'une Europe qui se projette vers l'Atlantique, en oubliant la Méditerranée, dont l'espace, l'histoire et la culture ont donné corps au code génétique de la civilisation occidentale ${ }^{12}$.

Et dans ce sens, «c'est bien dans la perte de centralité du mare nostrum que l'on pourrait retrouver les racines historiques, culturelles et sociales de la crise actuelle de l'Europe ${ }^{13}$. Néanmoins, dans la centralité même de son histoire, la Méditerranée a été aussi une Méditerranée d'exception, à savoir le lieu où s'est jouée une logique qui entrelace inclusion et exclusion et où les lignes de partage deviennent intérieures à ses espaces et même intimes aux corps qui les habitent. Depuis l'époque de la colonisation, comme le rappelle Barkat à propos de l'Algérie, la Méditerranée se constitue comme cette espace où les corps sont marqués, produits et institués selon des asymétries et des rapports de domination. Elle devient ainsi souvent l'espace de citoyennetés niées.

En conséquence de ces dynamiques de pouvoir et d'un type de «dispositif légal $»^{14}$ en vigueur sous la colonisation, les vies et les corps des colonisés sont destitués de la condition d'une «humanité pleinement réalisée, c'est-à-dire entièrement soumise au principe de raison ${ }^{15}$. Certains sujets sont donc jugés comme non conformes aux principes de la raison, à savoir externes aux cadres prétendument universels d'intelligibilité.

Ce que Barkat appelle «l'état de droit colonial » incarne alors une représentation emblématique de la façon dont les normes agissent pour rendre reconnaissables certains sujets et en exclure d'autres et, plus généralement, comme expression de cette logique que Butler décrit en tant que matérialisation des corps comme effet des dynamiques de 
pouvoir. En tant que constitué en relation avec un ordre normatif,

l'humain n'est pas seulement produit en opposition à l'inhumain, mais à travers un ensemble d'exclusions, d'effacements radicaux, auxquels est refusée, à strictement parler, la possibilité d'une expression culturelle. Par conséquent, il ne suffit pas d'affirmer que les sujets humains sont construits, car la construction de l'humain est une opération différenciatrice qui produit du plus et du moins humain $(\ldots)^{16}$.

Ce passage de Butler nous permet de fixer le noyau théorique de notre discours: des systèmes de genre aux systèmes raciaux et ethniques, l'humain est produit et institué par des pratiques discursives, régulatrices et des idéaux normatifs qui définissent ce qui est, à chaque fois, admis comme plus ou moins reconnaissable sur la scène publique. En effet, si «tout commence dans l'ordre du discours (...), certaines vies ne sont pas reconnues comme des vies (...), elles ne peuvent pas être humanisées, parce qu'elles ne rentrent dans aucun des cadres dominants qui définissent l'humain $(\ldots) »^{17}$. La culture et le discours sont donc des marqueurs qui forment et produisent ces corps et ces vies comme étant plus ou moins soumis à des critères établis d'intelligibilité.
Dans le cas de la colonisation cette logique semble être d'autant plus dure qu'elle opère à travers une naturalisation des frontières, à travers une ontologisation paradoxale et violente de la dynamique même de l'institution. Barkat analyse particulièrement un type d'institution, celle de l'indigénat, interne à l'état de droit colonial produisant une sous-catégorie d'individus dont le statut politique reste ambigu car, tout en faisant partie de la nation, ils n'y appartiennent jamais tout à fait, dans la mesure où ils ne jouissent pas pleinement des mêmes droits que ceux qui y sont pleinement admis. Ce qu'il appelle les «corps d'exception» sont donc ces corps constitués et matérialisés dans et par ce dynamisme pervers d'inclusion et d'exclusion qui les laisse comme suspendus dans une condition de perpétuel inaccomplissement d'une pleine citoyenneté. Ces corps et ces vies font donc partie de ceux qui ne comptent pas, de ceux dont l'inclusion dans le dispositif normatif qui les façonne ne les institue pourtant pas comme reconnaissables. Le corps de l'indigène et du colonisé est «contenu dans la société, inclus en tant que non compté, inclus en tant qu'exclu ${ }^{18}$. Et nous pourrions nous demander si cette logique paradoxale qui à la fois assimile et rejette n'est pas encore en acte aujourd'hui dans une certaine rhétorique universaliste qui cohabite avec 
ce que la sociologue féministe Christine Delphy a très justement qualifié de « systèmes de castes raciales » ${ }^{19}$, où la transmission du statut d'immigré, d'étranger se perpétuerait de génération en génération.

Or, il est important de comprendre comment un tel dispositif opère toujours à travers la naturalisation racialisante $\mathrm{du}$ groupe dominé, des 'étrangers' et plus généralement, des barrières qu'il institue entre les termes de la hiérarchisation. Les marqueurs de l'identité deviennent quelque chose de fixe, reconduit à une origine non modifiable garantissant, dès le départ et de manière stable, qui est capable et qui n'est pas capable de s'élever au niveau de la conscience morale et de transmettre l'héritage authentique et pur de la culture nationale.

L'image des corps indigènes soumis au régime d'exception - c'est-àdire l'image du corps d'exception - relève d'abord et avant tout du dispositif politique colonial avant d'avoir un quelconque rapport avec l'existence même des colonisés (...). C'est cette confusion perpétuée entre la réalité et son image étatique qui permet de se représenter un objet fabriqué (...) comme une réalité objective $^{20}$, donc de transformer une institution qui est toujours un produit historique en un donné naturel ou en une condition ontologique.
L'espace de la Méditerranée a donc été et quelque part continue d'être le lieu de l'institution de la différence en tant que fondée dans une présumée métaphysique des origines. Celle-ci entraîne l'érection de frontières imaginaires de l'identité nationale censées définir qui détient les droits de citoyenneté et qui en est exclu. La référence à une notion d'origine comme critère politique fondamental déterminant l'accès à la condition de citoyen a été un caractère du dispositif colonial. C'était bien l'appartenance à l'identité européenne, à une origine presque matricielle ou à une souche prétendument commune qui venait asseoir de tels droits. Ce qui est donc ici en jeu c'est une essentialisation de la différence qui vient s'inscrire dans la profondeur des corps de ceux et celles à qui on demande de s'uniformiser à des exigences et à des critères d'identité, de présumée authenticité nationale, sans leur donner pour autant les chances d'y parvenir ${ }^{21}$. L'indigène, comme le rappelle Barkat, est toujours soupçonné d'être foncièrement inadéquat à incarner les idéaux moraux et rationnels qui appartiennent à la filiation authentique de la nation. En réalité, une conception fausse de la civilisation fournit la mesure de l'humain et contribue corrélativement à produire un champ d'êtres aspirant à être reconnus comme humains, entretenus et détenus, condamnés 
à vivre et à mourir au sein d'une sphère extra-humaine et extra-juridique ${ }^{22}$.

Précisons brièvement la notion d'institution qui revient à plusieurs reprises dans le texte de Barkat: institution de l'indigénat, institution des corps en tant que corps d'exception exclus de la citoyenneté. Or, cette dimension de l'institution tend, dans la logique coloniale, à être occultée en tant que production du pouvoir. Cette logique prétend en effet ôter toute humanité aux corps des colonisés, les réduire à de purs corps organiques, les transformer en une «masse indigène $»^{23}$ dont les traits présumés altérés et pathogènes «se transmettraient ainsi de génération en génération $»^{24}$. Elle prétend de cette façon se prononcer sur leur intime statut naturel. En soulignant le caractère institué de ces corps et de la logique même qui les produit, nous mettons en avant le «processus de différencialisation et de naturalisation des rapports de pouvoir $»^{25}$ qui constitue et façonne les séparations qui sont à la base de toute idéologie raciale et nationaliste. Dès lors, la logique de la naturalisation qui érige et rigidifie les frontières entre les groupes est une institution, une production humaine ou un effet du pouvoir qui essaie pourtant de se faire passer pour un état objectif des choses.

\section{Ordre symbolique et transmission de la nation}

Là où il est question d'origines, d'une métaphysique essentialiste de l'identité qui fige le dynamisme même de l'histoire se pose aussi, de manière obsessionnelle et insistante, la question de la transmission de cette identité, des traits fondamentaux qui préalablement la caractériseraient. Nous avons d'ailleurs rappelé qu'une telle tentation identitaire a souvent traversé l'histoire des cultures de la Méditerranée qui se sont ainsi arrogées le privilège d'une quelconque authenticité et d'un héritage (moral, rationnel, religieux...) à transmettre en tant que non contaminé.

L'institution de l'indigénat constitue une tentative d'essentialiser la condition des colonisés à travers la représentation d'une postériorité, d'un héritage propre à la filiation indigène. Comme le souligne Barkat, une telle stratégie sert à créer et à maintenir une séparation entre le bon héritage de la nation authentique et la transmission de présumés traits pathogènes de la filiation indigène. En effet,

\footnotetext{
la transmission en question, où l'indigène recueille les caractères qui le particularisent comme une succession inéluctable - transmission que l'on peut
} 
dire héréditaire, en un sens particulier, non biologique - devient pourtant, à partir de l'identification de la nation vraie par la morale, la référence essentielle de la politique de défense de la souveraineté ${ }^{26}$.

Ce qui est en jeu c'est bien en effet une sorte de transmission culturelle, mais d'une culture presque naturalisée dans l'héritage prétendument pathologique et non modifiable de comportements voire d'inclinations jugés incompatibles avec les principes moraux et rationnels constituant l'ainsi nommée filiation authentique de la nation. Il nous semble alors que la transmission culturelle est ici réduite à une filiation qui se prétend «naturelle» qui vient reproduire un lien racialisé, «l'effet de l'altération des facultés subjectives », entendu comme un donné permanent au nom duquel les indigènes sont exclus de la citoyenneté.

Les travaux d'Elsa Dorlin ont, à ce propos, largement présenté et démasqué cette psychopathologie coloniale servant à 'légitimer' les rapports de domination: «le racisme est fondamentalement un régime psycho-pathogène $»^{27}$. C'est dans ce sens que, dans un texte consacré à Frantz Fanon, elle décrit la violence du régime colonial dans les termes d'un « 'monde sans médiation' (monde divisé, compartimenté, antagonique, frontal) $»^{28}$, traversé par des «mystifications (...) décelables dans la description clinique des pathologies psychiques des colonisés $»^{29}$.

A travers l'insistance sur la filiation, opère ainsi le fantasme des frontières indépassables de la nation qui se base sur le présupposé d'un lien essentiel, voire de sang entre ses membres. La référence insistante à l'origine et à sa prétendue pureté témoigne largement de cette logique.

D'ailleurs, encore aujourd'hui, le thème de la filiation demeure un point extrêmement sensible dans beaucoup de débats d'actualité sociale. Les inquiétudes autour de la transmission, d'une sorte de lien généalogique culturel voire national ne sont pas absentes de la scène politique et idéologique de nos jours. C'est ce que rappelle le sociologue Eric Fassin à propos $\mathrm{du}$ retour continuel sur les questions raciales, mais aussi à propos de ce qu'il appelle les questions sexuelles, à savoir les discussions polémiques sur les PaCS et sur les nouvelles parentalités. Fassin souligne, en effet, une «sacralisation française de la filiation $»^{30}$ qui implique pratiquement sa naturalisation comme façon de marquer les confins nets, presque racialisés, de la nation. De la fermeture du Pacs à une filiation (homosexuelle) au poids croissant de la référence à l'origine dans les politiques d'immigration, la filiation doit rester ancrée dans la nature, voire dans biologique. 
En effet, si l'on admettait que la filiation n'est qu'une convention, et non une donnée immuable, il serait plus malaisé de justifier sa fermeture. Tout se passe donc comme si l'enjeu majeur était bien de protéger la nationalité de toute “dénaturation".... 31 .

Dans la procédure de naturalisation on assiste de plus en plus à un basculement du droit de sol au droit de sang, où l'appel à la filiation biologique finit par primer. Le terme même de naturalisation ne présuppose-t-il pas déjà le paradoxe de devoir s'assimiler à une unité nationale fondée dans la nature, à savoir dans une origine commune et dans un lien de sang qui finit déjà par exclure tout se qui lui est étranger?

Si j'utilise ici le terme de naturalisation - explique Fassin -, c'est bien sûr pour marquer le paradoxe du droit de nationalité : naturaliser c'est rendre français; mais comment naturaliser si la francité est naturelle? L'étranger reste alors irrémédiablement étranger, malgré la naturalisation; mais du coup, le citoyen français 'de souche' se trouve naturalisé, dans un autre sens - biologique ${ }^{32}$.

La boucle de notre analyse est ainsi presque bouclée: ce paradoxe mis en évidence par Fassin dans la situation contemporaine était bien déjà à l'œuvre dans la logique des dispositifs coloniaux décrite par Barkat. Ce qui est en jeu, dans la pratique de la naturalisation, c'est la possibilité d'accéder à la citoyenneté. Néanmoins, pour les colonisés, leur origine joue comme un facteur qui empêche ou freine leur introduction dans le corps politique, car elle est perçue comme un élément dangereux pour l'intégrité de la nation.

La notion de filiation et les enjeux qui la caractérisent en termes de transmission de la culture nationale, de ses valeurs morales, juridiques ou rationnelles, certifiés et garantis par l'appartenance à une commune origine européenne, nous renvoie à un autre concept fondamental de l'unité nationale, à savoir l'ordre symbolique. Celui-ci devient un marqueur fondamental des frontières nationales voire des corps qui se trouvent soit inclus dans la nation soit confinés en une zone de non pleine intégration. Comme l'indique Barkat, la sphère politique semble ainsi presque se soumettre aux exigences de l'ordre symbolique et le gouvernement devient une façon de garantir et consolider la perpétuation de l'ordre moral. Ainsi la tâche de la politique consiste « à tracer les lignes de démarcation, à consolider les frontières imperméables, à construire des fortifications afin de s'assurer de la pérennité de l'ordre symbolique de la nation $»^{33}$.

Encore une fois, cette histoire ne s'arrête pas à l'époque de la colonisation ; 
elle traverse notre actualité et, en particulier, les inquiétudes autour de l'ordre symbolique et de ses possibles remaniements socio-historiques que l'on veut tenir à distance comme des formes de l'impossible et de l'impensable. Que se cache-t-il alors derrière cet ordre symbolique encore aujourd'hui si souvent évoqué dans maints débats brûlants qui touchent la famille, la sexualité et la nation? Pourquoi son maintien comme une structure intouchable et presque métahistorique, dans laquelle puisent les discours anthropologiques, psychanalytiques ou juridiques, a-t-il pu apparaître comme la garantie de stabilité de la culture et d'un ordre proprement humain du sens ? C'est Butler qui met en évidence le double registre de la notion de culture qui joue ici tant sur le plan anthropologique, comme une structure universelle d'intelligibilité, que sur le plan plus proprement national, où elle incarne l'héritage moral et rationnel de la tradition française. L'ordre symbolique fonctionne alors de pair avec l'ordre racialiste de reproduction de la culture et, dans le contexte français, de reproduction de l'identification implicite de la culture française et de l'universel (...). En ce sens, l'invocation de la loi symbolique agit ici comme une défense contre ce qui menace la pureté culturelle française, autrement dit les nouveaux modèles d'immigration, l'augmentation du nombre des métissages ou encore le brouillage des frontières nationales $^{34}$.

L'entrelacement profond qui lie, dans le contexte français, reproduction, filiation et transmission de la culture éveille des fantasmes de pureté qui conduisent à la quête d'un horizon de normes prétendument stables et non modifiables qui garantissent la pérennité du lien identitaire de la nation. En ce sens, Butler identifie tant dans les nouveaux phénomènes liés à l'immigration et au métissage que dans les débats sur les changements qui affectent l'ordre classique de la famille et de la filiation (PaCS, procréation médicalement assisté, nouvelles parentalités non nécessairement liées au cadre hétérosexuel) les éléments qui viennent questionner profondément et même bouleverser l'ordre culturel, symbolique voire racial censé fonder la nation.

Pour bien comprendre ce point, il est nécessaire d'examiner comment opère le terme 'culture' et comment, dans le contexte des débats français sur l'immigration, le $\mathrm{PaCS}$ et l'homoparentalité, ce terme a été invoqué non pour désigner les formes culturelles variables de la vie humaine, mais les conditions universelles de l'intelligibilité humaine ${ }^{35}$. 
Plus précisément, l'universalisme culturel de l'ordre symbolique incarne presque paradoxalement un caractère spécifique de la culture française. L'universalisme républicain qui caractérise, comme le dit non sans ironie Delphy, «l'exception française $»^{36}$, apparaît comme un trait de l'identité nationale. Derrière la présomption d'universalisme qui refuse de prendre en compte les questions minoritaires dans la scène publique se cache alors la négation des discriminations voire des fractures internes qui traversent encore bien aujourd'hui la société française. L'égalité reste ainsi plutôt quelque chose de formel, car toute revendication de reconnaissance de la part de ces «autres» (ceux qui ne sont pas «nous») est ressentie comme incompatible avec les principes de l'universalisme, donc pour reprendre Delphy, avec une certaine représentation que la nation se fait d'elle-même.

On a donc affaire à un champ schématique où les mots (..) 'communautarisme', 'maghrébin', 'musulman', 'immigré', voire ‘immigré de deuxième génération' forment un ensemble dans lequel chacun peut être utilisé pour signifier l'autre; cet ensemble forme un couple avec l'ensemble 'république', 'intégration', 'nation', 'identité nationale' (...). Ces couples d'opposition servent à justifier de façon acceptable une définition ethnique de la nation française, puisque république, dans la configuration française, est interchangeable avec 'nation, 37 .

Le problème c'est que l'origine compte encore souvent dans la définition de la citoyenneté et que la condition d'étranger ou d'immigré est transformée en une condition permanente qui se transmet de génération en génération en donnant lieu à une descendance immigrée. Il $\mathrm{y}$ a donc ici une essentialisation racialisée qui fige la place de ceux qui sont perçus comme des éternels arrivants et, comme tels, « destinés à quitter le territoire français $»^{38}$. Ici aussi on se trouve face à une conception de la filiation qui fixe l'autre dans sa différence en le reliant à la position d'étranger presque sans issue. De cette manière, le principe de la différence que l'universalisme républicain avait refoulé en tant que formulation culturaliste voire racialisante, est réintroduit soudainement dans le cœur même de la position universaliste. Plus précisément celle-ci semble se renverser en ce qu'elle avait cru être son opposé, le langage différentialiste de la racialisation.

En effet, comme le dit Fassin, la question est bien plus complexe que le schéma qui oppose un universalisme prétendument anti-identitaire et un différentialisme communautariste et conséquemment raciste : ainsi «l'émergence d'une politique 
minoritaire ${ }^{39}$ apparaitt aujourd'hui de plus en plus «définie en termes de discrimination plutôt que d'identité ${ }^{40}$, donc en termes de dénonciation des inégalités réelles et effectives qui trop souvent se cachaient derrière l'affirmation d'une égalité abstraite et formelle.

$\mathrm{Si}$ dès lors «l'alternative entre universalisme républicain et différentialisme communautariste $»^{41}$ s'avère être de moins en moins crédible, l'apport des études post-coloniales nous aura-t-il aidé à ébranler les certitudes d'une histoire méditerranéenne encore trop souvent racontée voire dominée par un seul des deux bords ?

\section{Conclusion}

Nous voudrions reprendre les réflexions de R. Ivekovic au colloque de Catane et particulièrement sa critique de toute image édulcorée de la Méditerranée et de son histoire qui en ferait un lieu «métisse et hybridé (...) de croisements mystifiés dans la célébration des différences $»^{42}$. R. Ivekovic nous met donc en garde contre une telle image simpliste qui risquerait trop facilement d'oublier que la Méditerranée est également traversée par bien des inégalités et des asymétries. En particulier, cette perspective mystifiée, toute européenne, car sa « vérité » apparaît difficilement partageable de l'autre bord, présente les difficultés «inhérentes à toute construction humaine régie par une intention universalisante $»^{43}$. En effet, précise encore Ivekovic,

\begin{abstract}
l'universel dénonce toujours sa provenance d'une asymétrie qui se veut néanmoins neutre, et d'une hiérarchie plus ou moins bien imposée ou même acceptée. En tout cas, les particularités qui demeurent à la base de l'universel ne sont pas égales, ni dans leurs rapports à la liberté, ni dans leurs rapports réciproques ${ }^{44}$.
\end{abstract}

Comment rompre alors les représentations dominantes et les rapports de pouvoir contenus dans la logique d'un universel encore pourtant si particulier? En conclusion et pour essayer d'ébaucher une réponse à cette question, nous voudrions reprendre un aspect qui aurait mérité un plus vaste développement (et que nous n'avons pu aborder que de façon marginale): la prise en compte de «la multiplicité des oppressions et de leurs combinaisons $\gg{ }^{45}$ telles qu'elles se sont jouées et continuent de se jouer sur les bords de la Méditerranée. Car la Méditerranée a souvent été érigée comme barrière ou frontière marquant des formes de domination différentes: le cas du sexisme en est l'exemple le plus éloquent. Il s'agit alors de montrer que ces formes d'asymétrie et d'inégalité, qu'on présente encore bien souvent comme segmentées, 
partagées dans l'espace méditerranéen sont entrelacées, articulées selon les termes de race, classe, sexe/genre. Outre le fait qu'il rend possible une lecture plurielle et complexe permettant de démasquer les rapports de forces, les formes d'oppression et de discrimination dans leurs multiples aspects, ce paradigme, qu'on nomme souvent intersectionnalité, nous semble aussi très prometteur dans la mesure où il permet de rompre la clôture identitaire et la naturalisation des groupes sociaux en montrant comment les logiques de la domination les traversent et les parcourent à divers degrés.

Il faut donc penser - affirme Delphy - la spécificité de l'oppression de 'race' : de l'oppression des indigènes, sans oublier, sauf à nos risques et périls, que cette population rassemblée par une oppression est divisée par une autre. Ce n'est pas nous qui la divisons ou la diviserons :

elle est toujours déjà divisée. C'est pourquoi je considère que lutter contre l'oppression de genre en son sein et tout de suite n'est pas un luxe, mais la condition sine qua non pour une lutte efficace ${ }^{46}$.

Or, pour Delphy, l'inverse est aussi valable: aucune véritable lutte contre l'oppression de genre, aucune lutte féministe (ou anti-homophobe) ne pourra faire l'impasse sur le racisme et céder à la tentation de culturaliser voire de racialiser l'une ou l'autre forme de domination (notamment la domination sexiste et homophobe) en en faisant la spécificité d'un groupe et en la repoussant de l'autre côté de la barrière.

\section{Notas}

${ }^{1}$ P. BARCELLONA - F. CIARAMELLI (sous la dir. de), La frontiera mediterraneea. Tradizioni culturali $e$ sviluppo locale, saggi introduttivi, il Dedalo, Bari, 2006, p. 6.

${ }^{2}$ Ibidem, p. 7.

${ }^{3}$ Par études post-coloniales on entend un courant de pensée qui s'est développé aux Etats-Unis pendant les années 1980 et qui s'installe de plus en plus dans les départements de littérature et de sciences sociales. Elles s'intéressent aux formes de domination et aux rapports de pouvoir, notamment ceux qui dérivent de la colonisation et de son héritage (processus de colonisation et de décolonisation) et mettent en question le primat de l'européocentrisme et de la pensée occidentale. En affirmant une perspective post-structuraliste et constructiviste, les études post-coloniales contestent toute essentialisation des phénomènes sociaux et culturels. Ceux-ci sont plutôt des produits historiques en devenir, toujours hybrides et pluriels. L'attention qu'elles portent aux 
groupes minoritaires, aux «sans voix » et aux groupes discriminés les rend proches des études subalternes. En France, le développement, encore assez limité, de ces études est lié à certaines réflexions autour des émeutes des banlieues et au débat sur le port du voile où est menée une critique de l'universalisme républicain aveugle aux discriminations dont souffrent les groupes ethnicisés voire racialisés, à savoir les descendants d'immigrés maghrébins et africains.

${ }^{4}$ R. IVEKOVIC, « Ragione con-divisa, tra passione e ragione », dans La frontiera mediterranea. Tradizioni culturali $e$ sviluppo locale, p. 63-72, p. 70.

${ }^{5}$ Ibidem, p. 65.

${ }^{6}$ P. BARCELLONA - F. CIARAMELLI (sous la dir. de), La frontiera mediterraneea. Tradizioni culturali $e$ sviluppo locale, saggi introduttivi, op. cit. p. 11.

${ }^{7}$ Ibidem, p.12.

${ }^{8}$ S. M. BARKAT, Le corps d'exception. Les artifices du pouvoir colonial et la destruction de la vie, Ed. Amsterdam, 2005, p. 10.

${ }^{9}$ Ibidem, p. 12.

${ }^{10}$ J. BUTLER, Trouble dans le genre. Le féminisme et la subversion de l'identité, La Découverte, Paris, Paris, 2005, p. 245.

${ }^{11}$ Ibidem, p. 61.

${ }^{12}$ P. BARCELLONA - F. CIARAMELLI (sous la dir. de), La frontiera mediterraneea. Tradizioni culturali $e$ sviluppo locale, saggi introduttivi, op. cit. p. 9.

${ }^{13}$ Ibidem, p. 10.

${ }^{14}$ S. M. BARKAT, Le corps d'exception. Les artifices du pouvoir colonial et la destruction de la vie, ibidem, p. 70.

${ }^{15}$ Ibidem, p. 20.

${ }^{16}$ J. BUTLER, Ces corps qui comptent. De la matérialité et des limites discursives du sexe, Ed. Amsterdam, Paris, 2009, p. 21.

${ }^{17}$ J. BUTLER, Vie précaire. Le pouvoir du deuil et la violence après le 11 septembre 2001, Ed. Amsterdam, 2005, p. 61. La notion de cadre est très importante dans la réflexion de Butler autour du pouvoir et de son fonctionnement concret opérant à travers des critères précis d'intelligibilité. «Donc, quand on parle de cadres (Frameworks) dans ce contexte, on ne parle pas seulement des perspectives théoriques que nous mobilisons pour analyser la politique, mais de modes d'intelligibilité qui concourent au fonctionnement de l'Etat et qui, en tant que tels, sont eux-mêmes des exercices de pouvoir, alors même qu'ils excèdent le domaine spécifique du pouvoir d'Etat », J. BUTLER, Ce qui fait une vie. Essai sur la violence, la guerre, le deuil, Zones, Paris, 2010, p. 144.

${ }^{18}$ S. M. BARKAT, Le corps d'exception. Les artifices du pouvoir colonial et la destruction de la vie, ibidem, p. 72. 
${ }^{19}$ C. DELPHY, Classer, dominer. Qui sont les 'autres'?, La Fabrique, Paris, 2008, p. 139. la notion de caste vient complexifier le concept de racisme soulignant la spécificité de l'oppression de race en relation avec l'oppression de classe. Ce que la notion de caste rajoute c'est donc le caractère prétendument statique d'une catégorie sociale sur la base d'un principe de transmission presque biologique. En effet, «quand on hérite exactement $d u$ statut de ses parents, sans mobilité probable ni même possible, il ne s'agit plus d'une situation de classe, mais d'une situation de caste. C'est ce qui est en train de se créer en France. Et le langage l'indique: on parle d'immigrés de deuxième génération voire de troisième génération; on transforme la situation, par définition temporaire, d'immigré, en caractère héréditaire et quasi biologique », (ibidem, p. 146-147).

${ }^{20}$ S. M. BARKAT, Le corps d'exception. Les artifices du pouvoir colonial et la destruction de la vie, op. cit. p. 43.

21 Nous faisons ici référence à ce que Delphy appelle la logique du double bind qui est ici propre au système des castes raciales dont l'essentialisation rend les frontières entre les groupes presque imperméables. «Cette population est prise dans un redoutable double bind: on la somme de se montrer 'pareille', mais on la perçoit et on la nomme 'différente'.
Quoiqu'ils ou elles fassent, au terme du cursus, elles et ils échouent toujours à l'examen, elles et ils n'arriveront jamais à satisfaire les critères de francité. Car la réalité non dite, c'est que ces critères excluent par définition toute personne d'origine maghrébine ou africaine », (C. DELPHY, Classer, dominer. Qui sont les 'autres'?, op. cit. p. 149). Delphy insiste sur le fait que cette logique coloniale traverse bien l'actualité post-coloniale française qui n'a pas encore surmonté les catégorisations et les discriminations cachées derrière la rhétorique de l'égalité formelle propre à son universalisme.

${ }^{22}$ J. BUTLER, Vie précaire. Le pouvoir du deuil et la violence après le 11 septembre 2001, op. cit. p. 123.

${ }^{23}$ S. M. BARKAT, Le corps d'exception. Les artifices du pouvoir colonial et la destruction de la vie, op. cit. p. 40.

${ }^{24}$ Idem.

25 E. DORLIN, La matrice de la race. Généalogie sexuelle et coloniale de la Nation française, La Découverte, Paris, 2006, p. 11. Cette référence aux thèses de Dorlin nous fait peut-être nous déplacer quelque peu par rapport à la centralité méditerranéenne de notre discours sur les rapports de domination coloniale. Elle situe en effet la généalogie de l'idéologie nationale et raciale française dans le cadre de la colonisation des Caraïbes et des Antilles à l'âge classique. C'est dans ce 
contexte qu'est introduite la notion d'indigène comme ne faisant pas partie du corps social dont découle la souveraineté. En particulier, souligne Dorlin, l'introduction de la notion d'un tempérament naturel commun de la classe des planteurs a permis de définir la nation au sens nouveau. En tant qu'il opère une naturalisation des différences, «le tempérament peut être défini comme un outil politique privilégié, qui permet de naturaliser la nation : il fait de la nation une entité dont l'identité est présente et se développe en chacun », (ibidem, p. 198).

${ }^{26}$ S. M. BARKAT, Le corps d'exception. Les artifices du pouvoir colonial et la destruction de la vie, op. cit. p. 40.

${ }^{27}$ E. DORLIN, «Je ne suis pas esclave de 1'Esclavage. Corps, violence et subjectivité chez Frantz Fanon », dans Geste, n. 6 / 2009, p. 298-307, p. 306.

${ }^{28}$ Ibidem, p. 305.

${ }^{29}$ Idem.

${ }^{30}$ E. FASSIN, « Naturalisations. Filiation, famille et nationalité », dans Reproduire le genre, Bibliothèque du Centre Pompidou, 2010, p. 137-143, p. 137.

31 E. FASSIN, «Questions sexuelles, questions raciales. Parallèles, tensions et articulations », dans D. FASSIN et E. FASSIN, De la question sociale à la question raciale. Représenter la société française, La Découverte, Paris, 2006, 2009, p. 238-256, p.246.
${ }^{32}$ E. FASSIN, «Naturalisations. Filiation, famille et nationalité », dans Reproduire le genre, op. cit. p. 141.

${ }^{33}$ S. M. BARKAT, Le corps d'exception. Les artifices du pouvoir colonial et la destruction de la vie, op. cit. p. 41.

34 J. BUTLER, Défaire le genre, Ed. Amsterdam, 2006, p. 144.

${ }^{35}$ Ibidem, p. 140.

36 C. DELPHY, Un universalisme si particulier. Féminisme et exception française (1980-2010), Syllepse, Paris, 2010, p. 8.

${ }^{37}$ Ibidem, p. 201.

${ }^{38}$ C. DELPHY, Classer, dominer. Qui sont les 'autres'?, op. cit. p. 146.

${ }^{39}$ E. FASSIN, «Aveugle à la race ou au racisme ? Une approche stratégique », dans D. FASSIN et E. FASSIN, De la question sociale à la question raciale, op. cit. p. 114-138, p. 134.

${ }^{40}$ Idem.

${ }^{41}$ Idem.

${ }^{42}$ R. IVEKOVIC, « Ragione con-divisa, tra passione e ragione », dans La frontiera mediterranea. Tradizioni culturali $e$ sviluppo locale, op. cit. p. 66.

${ }^{43}$ Idem.

${ }^{44}$ Idem.

${ }^{45}$ C. DELPHY, Classer, dominer. Qui sont les autres?, op. cit. p. 172.

${ }^{46}$ Ibidem, p. 173. 


\section{Referências}

Barcellona, P. Ciaramelli, F. (2006), La frontiera mediterraneea. Tradizioni culturali e sviluppo locale. Bari: Dedalo.

Barkat, S.M. (2005) Le corps d'exception. Les artifices du pouvoir colonial et la destruction de la vie. Paris: Ed. Amsterdam.

Butler, J. (2005) Trouble dans le genre. Le féminisme et la subversion de l'identité. Paris : La Découverte.

Butler, J. (2005) Vie précaire. Le pouvoir du deuil et la violence après le 11 septembre 2001. Paris: Ed. Amsterdam.

Butler, J. (2006) Défaire le genre. Paris : Ed. Amsterdam.

Butler, J. (2009) Ces corps qui comptent. De la matérialité et des limites discursives $d u$ sexe. Paris: Ed. Amsterdam.

Butler, J. (2010) Ce qui fait une vie. Essai sur la violence, la guerre, le deuil. Paris : Zones.

Delphy, C. (2008) Classer, dominer. Qui sont les 'autres'?. Paris: La Fabrique.

Delphy, C. (2010), Un universalisme si particulier. Féminisme et exception française (1980-2010). Paris : Syllepse.

Dorlin, E. (2006) La matrice de la race. Généalogie sexuelle et coloniale de la Nation française. Paris : La Découverte.

Dorlin, E. (2009) «Je ne suis pas esclave de l'Esclavage. Corps, violence et subjectivité chez Frantz Fanon», Geste, 6, 298-307

Fassin, E. (2009) «Questions sexuelles, questions raciales. Parallèles, tensions et articulations », dans Fassin, D. et Fassin, E. De la question sociale à la question raciale. Représenter la société française ( $p$ 2 238-256). Paris : La Découverte.

Fassin, E. (2009) « Aveugle à la race ou au racisme? Une approche stratégique », dans Fassin, D. Fassin, E. De la question sociale à la question raciale (pp 114-138). Paris : La Découverte.

Fassin, E. (2010) «Naturalisations. Filiation, famille et nationalité », dans Reproduire le genre (pp. 137143). Paris : Bibliothèque du Centre Pompidou.

Ivekovic, R. (2006) «Ragione con-divisa, tra passione e ragione » dans $L a$ frontiera mediterranea. Tradizioni culturali e sviluppo locale, (pp. 6372). Bari: Dedalo.

Catarina Rea: Graduação em Letras e Filosofia pela Universidade Sacro Cuore de Milão (Itália). Doutorado em Filosofia 
pela Université Catholique de Louvain (2007). Chargé de cours - Université Lille 3 - Sciences Humanes, Arts et Culture. Pós Doutoranda na UFSC.

e-mail: caterina.rea@neuf.fr 